\title{
Correction to: Improved model for studying hydrological process in the field-to-field irrigation system
}

\author{
Arash Ebadi $^{1} \cdot$ Omid Raja ${ }^{1} \cdot$ Hamed Ebrahimian ${ }^{1}$ (I) Mohammad Reza Yazdani ${ }^{2} \cdot$ Vahid Rezaverdinejad $^{3}$
}

Published online: 3 March 2022

(c) The International Society of Paddy and Water Environment Engineering 2022

Correction to: Paddy and Water Environment

https://doi.org/10.1007/s10333-021-00882-3

In the original publication of this article, the title was incorrectly given as Improved model for studying hydrological process in the field-to-filed irrigation system but should have been Improved model for studying hydrological process in the field-to-field irrigation system. It has been updated in this correction.

The original article has been corrected.

The original article can be found online at https://doi.org/10.1007/ s10333-021-00882-3.

Hamed Ebrahimian

ebrahimian@ut.ac.ir

1 Department of Irrigation and Reclamation Engineering,

University of Tehran, Karaj, Iran

2 Rice Research Institute of Iran, Agricultural Research, Education and Extension Organization (AREEO), Rasht, Iran

3 Department of Water Engineering, University of Urmia, Urmia, Iran 